\title{
Problematizzazione, contesa e rivalutazione del bello nell'arte, tra relativismo filosofico, pratica artistica e senso comune
}

\author{
di Nicola Vitale \\ ex@nicolavitale.com
}

\begin{abstract}
Aesthetic perception is today a confused and controversial experience. In common sense relativistic conception of beauty, coexists with the consideration of the so-called "masterpieces" as works in which there is a stable aesthetic value. Philosophical and scientific relativism seems to have definitively set aside the conception of beauty not only as a universal value, but also as the essence of art, as it is counted among those universal metaphysical values, which have long been questioned. But some philosophers, such as Severino, say the opposite. Today seems to be a tendency to rediscover beauty above all in art, as a contemplative perception. Would the eventual return of art to beauty mean a return to universal metaphysical values? The difference between Kantian adherent beauty and free beauty is analyzed. The first is linked to metaphysical values, as an expression of an idea. The second, free beauty, on the other hand, has no metaphysical characteristics because it is not linked to a concept, therefore an expression of empirical harmonies. But also with regard to free beauty, the Kantian idea that sentiment can perceive its universality as an intersubjective value, is today difficult to accept both theoretically and empirically. This happens because today sentiment is no longer cultivated in the perception of beauty through canons, which are also disqualified for the pretense of universality in determined forms. Here, too, a distinction must be made between classical anthropometric canons and archaic non-naturalistic canons. We discover that the former are affected by a metaphysical foundation, while the latter reveal a different structure with other functions. According to Florenskij, the canon is not oppressive but liberating. On these suggestions and on empirical evidence we theorize that the (non-naturalistic) canon constitutes a guide for the recognition of a polyvalence of expressive language in which feeling coordinates with the other functions of consciousness, leading to transcend language itself in a non-metaphysical dimension. This suggests that this polyvalent structure that emerges from the canons is associated with beauty, as aimed at its realization.
\end{abstract}

Keywords: Aesthetic perception, Relativism, Beauty, Canon

Materiali di Estetica - N. 8.2: 2021, Pagina 381 


\section{Attuale concezione del bello rispetto all'arte}

C'è chi frequenta musei in cerca di un piacere superiore davanti a capolavori della pittura e della scultura. Coloro che sensibili alle forme espressive ascoltano musica, leggono romanzi e poesie, frequentano teatri e cinema per sentire verificarsi in loro quella particolare sintesi estetica che chiamiamo "bello".

Frequentemente tra questa stessa umanità si sente ripetere che il bello è una percezione "soggettiva". Come abbiamo anche sentito ripetere da tempo che, essendo il bello una questione inerente l'edonismo individuale, il senso autentico dell'arte è qualcosa di più profondo e articolato. L'influenza sul senso comune della cultura contemporanea è evidente: la storia dell'arte, l'estetica, la filosofia e teoria delle arti, oggi prevalentemente negano all'arte il suo senso e valore più immediato, in nome di un relativismo salutare, come esito estremo di quella critica ai valori universali metafisici che arriva da lontano, che nel Novecento si è consolidata.

Attribuendo al bello una valenza "soggettiva", si richiama evidentemente una proposizione kantiana della Critica del Giudizio ${ }^{1}$ (ed è forse per questo che la convinzione ha assunto tale autorevolezza). Ma Kant, se è vero che dice qualcosa di simile, lo intende in altro modo da come è espresso e inteso nel senso comune, in cui con "soggettivo" si intende relativo, non universale. Kant invece sostiene che il giudizio di gusto (come facoltà di percepire il bello) è un giudizio riflettente, soggettivo, universale. Cioè sostiene che il bello non è nell'oggetto, ma è il soggetto che interpreta la particolare conformazione dell'oggetto come bello. Ma Kant sostiene anche che tutti i soggetti hanno la stessa facoltà di gusto, cioè di cogliere il bello, che dunque risulta un valore condiviso, intersoggettivo e universale.

\footnotetext{
${ }^{1}$ Cfr. I. Kant, Critica del Giudizio (1790), tr. it. a cura di L. Amoroso, Rizzoli, Milano 1995, pp. 175 e ss.
} 
Peraltro tra i filosofi prevale la convinzione, non solo che il bello sia un fattore relativo dell'arte, ma anche secondario, cosa che sembrerebbe avallata dallo stesso Kant quando, pur ammettendo che il bello è un valore universale, afferma che una percezione più profonda dell'arte implica funzioni intellettuali, portando a "pensare molto», spostando il focus dell'arte nel pensiero. Evidentemente questo "pensare" è riferito a concetti indeterminati, espressi in una dimensione simbolica, quanto però basta a Kant per sancire il primato della ragione ${ }^{2}$.

Sulla scorta di tali diffuse convinzioni, coloro che amano l'arte continuano a frequentarla, tuttavia per non sentirsi "superficiali edonisti", cercando motivi diversi, ascoltando le audioguide nei musei, leggendo testi esplicativi. Ma traditi poi dai loro stessi giudizi sulle opere, che vengono infine valutate come belle o brutte, e non certo come interessanti o istruttive o semplicemente valide. Una riprova è nel fatto che generazioni sono ripetutamente affascinate sempre dalle stesse opere intramontabili, che vengono definite "capolavori". Il concetto stesso di capolavoro designa una gerarchia qualitativa delle opere, stabilita da lungo tempo in un dibattito trans-storico, quale gerarchia stabile della qualità estetica, cioè del bello. Diversamente usciremmo dalla dimensione estetica, per cui qualsiasi documento storico potrebbe competere con un'opera d'arte, anche per quel «pensare molto» kantiano.

Questa confusione nel rapporto tra arte e bello sembra nascere da una contrapposizione tra istinto e cultura, una cultura che si oppone all'istinto estetico per portare su di un piano diverso il senso dell'arte, che si vorrebbe, come tutto il resto, sottomesso alla ragione e alla scienza.

Oggi tuttavia si avverte emergere, in modo sempre più marcato, la questione del bello come necessità di liberarsi di tali premesse o "pregiudizi",

\footnotetext{
${ }^{2}$ In Kant la caratteristica del genio è «facoltà di esibizione delle idee estetiche; dove per idee estetiche intendo quelle rappresentazioni dell'immaginazione, che danno occasione a pensare molto, senza che però un qualunque pensiero o un concetto possa essere loro adeguato». I. Kant, Critica del Giudizio, cit., pp. 108, 177.
} 
che dir si voglia, per godere dell'arte ricominciando dalla dimensione contemplativa, questione che come vedremo apre a problematiche generali rilevanti.

Occorre dunque più che mai interrogarsi sul bello, in particolare sul bello artistico, chiedendosi di cosa si tratta, ma soprattutto se il tornare ad accettare l'universalità del bello come essenza dell'arte, debba necessariamente comportare il ritorno ai vecchi valori metafisici, così a lungo controversi perché ritenuti abusivi.

\section{Un punto di vista pratico sull'arte}

Qui si spalanca una vasta questione, che se affrontata non solo dal punto di vista filosofico-estetico, ma anche da un punto di vista empirico, legato alla conoscenza sul campo dei valori sensibili dell'arte, potrebbe fare più luce.

Vico, nel Seicento, sosteneva che per conoscere una cosa bisogna saperla fare $^{3}$. Nietzsche, nell'Ottocento, affermava che l'arte dovesse essere vista dalla prospettiva dell'artista ${ }^{4}$. Valéry, nel primo Novecento, fa un appello ai filosofi sostenendo che occorresse tornare ad affinare la percezione delle opere per evitare uno sterile intellettualismo ${ }^{5}$. Più recentemente sono diversi i filosofi e studiosi che si sono avvicinati a questa esigenza di "presa diretta" della percezione estetica, come ad esempio Wittgenstein che sosteneva che dell'arte non bisognasse parlare e quindi lasciarla nelle mani di chi la fa e la fruisce $^{6}$. O ancora George Steiner che in Vere presenze (1989) denuncia una cultura del commento, che aveva preso il sopravvento sulla percezione diretta delle opere ${ }^{7}$.

${ }^{3}$ Cfr. G. Vico, De antiquissima Italorum sapientia (1710), in Opere filosofiche, a cura di P. Cristofolini, Firenze, Sansoni 1971.

${ }^{4}$ Cfr. M. Heidegger, Nietzsche, tr. it. a cura di F. Volpi, Adelphi, Milano 1994, p. 80.

${ }^{5}$ P. Valéry, Discours sur l'esthétique, in Euvres, 2 voll., Gallimard, Paris 1975-1977, I, p. 24.

${ }^{6}$ Cfr. L. Wittgenstein, Tractatus logico-philosophicus, tr. it. a cura di G. C. M. Colombo, Fratelli Bocca Editori, Roma-Milano 1954, proposizione n. 7.

${ }^{7}$ Cfr. G. Steiner, Vere presenze, tr. it. di C. Béguin, Garzanti, Milano 1992. 
L'estetologo Dino Formaggio, alla fine del Novecento, sentiva che per poter parlare dell'arte in termini fenomenologici dovesse sporcarsi le mani, cioè provarsi in una disciplina artistica, affrontando così la scultura e facendone una sua seconda attività8.

Ma proprio questa posizione di Formaggio deve farci riflettere sulla questione attuale circa l'arte e il bello, tra teoria e pratica. Infatti occorre chiedersi come Formaggio intendesse il lavoro dello scultore e, di riflesso, quello dell'artista in genere. Avendo ormai la nostra cultura abbandonato i canoni di ogni tradizione, l'arte da tempo è entrata in un relativismo estenuato dove tutto è possibile. Così, nell'intraprendere l'attività di scultore Formaggio, pur convinto di "fare esperienza", si dedica alla disciplina secondo una concezione dell'arte niente affatto originaria e ingenua, ma preconizzata da un particolare sguardo teoretico. In effetti, tra il ventaglio delle molte interpretazioni dell'arte, sembra prediligere il concetto di metis, come intelligenza empirica, capacità di proporre soluzioni concrete ai casi specifici, una immaginazione creativa in grado di trasformare gli oggetti, per cui nelle lezioni in Università, come riportato nei suoi testi, poneva come esempio mitologico la metamorfosi operata da Hermes della tartaruga in lira, così come si riferiva spesso alla Testa di toro di Picasso (1942), realizzata assemblando la sella e il manubrio di una bicicletta da corsa ${ }^{9}$.

\section{Che cos'è l'arte? Tra relativismo e statistica}

Il caos che pervade l'arte contemporanea, cui abbiamo accennato, emerge efficacemente dal libro L'arte espansa di Mario Perniola (2015), dove confronta recenti Biennali di Venezia ${ }^{10}$. Qui il filosofo rileva come oggi l'élite culturale, nell'ambito delle arti visive (o visuali) consideri arte tutto e il

\footnotetext{
8 G. Scaramuzza, "Dino Formaggio: una presentazione", Eikasia, Revista de Filosofía, LXII, 2015, pp. 13-28.

${ }^{9}$ Cfr. D. Formaggio, L'arte come idea e come esperienza, Morcelliana, Brescia 2018.

10 M. Perniola, L'arte espansa, Einaudi, Torino 2014.
} 
contrario di tutto. Un libro peraltro ironico, come era lo spirito del filosofo, che sfocia in tratti comici, ad esempio affermando che nel futuro non saranno più i critici a stabilire cosa sia arte, ma gli psichiatri. D'altro canto, dopo che negli anni Sessanta il pittore Pierre Brassau, proposto da una galleria svedese, raggiunto un certo successo, si è rivelato essere uno scimpanzé, subito emulato da muli, elefanti e affini, il relativismo transumano dell'arte appare evidente.

Emerge il fantasma del detto di Bruno Munari: «Quando tutto è arte, niente è arte» ${ }^{11}$. Questo è il rischio della situazione attuale.

Ma cosa affermava Formaggio a proposito? "Arte è tutto ciò che gli uomini chiamano arte»12. Un'affermazione apparentemente tautologica, ma che richiama la concezione kantiana di intersoggettività. Non potendo esistere una definizione razionale del bello e dell'arte, queste vengono legittimate dalla collettività, da cui dovrebbe emergere un giudizio condiviso.

Vi è tuttavia una differenza, in quanto se Kant vedeva l'arte necessariamente legata al bello e al sublime, Formaggio pensa a un'arte indipendente dal bello. Nel Novecento tra i filosofi che identificavano l'arte col bello troviamo Emanuele Severino, che scrive: «L'arte e la bellezza (sono sinonimi perché un'arte non bella è un'arte non riuscita, una non arte) sono rimedi $[\ldots] »^{13}$. Tuttavia né Kant, né tanto meno Severino, pensavano che il bello, come essenza dell'arte, avrebbe dovuto richiamare valori universali metafisici promulgati innanzitutto da Platone, che vedeva il bello come una manifestazione sensibile dell'idea. Un tema importante che riprenderemo in seguito.

Ma tornando alla concezione dell'arte (più o meno legata al bello), la cui essenza viene affidata da Formaggio alla sensibilità della collettività,

\footnotetext{
${ }^{11}$ Cfr. B. Munari, Munari 80: a un millimetro da me. Teoremi, brustoline e disegni al telefono, Scheiwiller, Milano 1987.

12 D. Formaggio, L'arte come idea e come esperienza, Mondadori, Milano 1981, p. 11.

${ }^{13}$ E. Severino, Il bello, Mimesis, Milano-Udine 2011, p. 22.
} 
osserviamo che egli non considera che, in tale clima culturale, dove qualsiasi concezione dell'arte era (ai suoi tempi, come oggi) possibile, "ciò che gli uomini chiamano arte" sia un giudizio che difficilmente può convergere in un responso unitario. In tale mare magnum forse l'unica possibilità di orientarsi è aggiungere all'ontologia dell'arte di Formaggio un numero di percentuale. In questo modo avremmo una statistica e una graduatoria. Facendo delle ipotesi: La Monna Lisa di Leonardo potrebbe essere arte al 99,7\%, Guernica di Picasso all'82\%, Marilyn di Andy Warhol 33\%, lo squalo in formaldeide di Damien Hirst 2,1\%.

Ma se generalizziamo e allarghiamo questo confronto alla totalità degli uomini, e non solo a qualche sparuto consesso di specialisti, intellettuali, nostalgici o manici delle stampe giapponesi o altro, scopriamo che la stragrande maggioranza degli uomini e donne che si interessano di arte, hanno fatto una scelta netta su cosa oggi sia da considerarsi arte, che ci permette di trarre delle conclusioni.

Infatti tanto quanto i musei di arte antica e moderna straripano di visitatori, così i musei di arte contemporanea sono del tutto vuoti, nonostante siano passati ormai sessant'anni dalla sua fondazione. Per questa questione rimando a due libri di Adriana Polveroni: This is contemporary! Come cambiano i musei d'arte contemporanea (2007), Lo sboom (2009)14. Una studiosa che ha fatto da più di un decennio un'indagine proprio sui musei di arte contemporanea, arrivando alla conclusione che l'arte contemporanea non ha pubblico.

La prima deduzione che possiamo trarre da questo dato di fatto è che la maggior parte del pubblico generico non considera l'arte contemporanea "arte", o potrebbe anche essere che, pur considerandola arte, la ritiene difficile, indigesta, noiosa, insomma non degna di essere presa in

${ }_{14}$ Cfr. A. Polveroni, This is contemporary! Come cambiano i musei d'arte contemporanea, FrancoAngeli, Milano 2007; A. Polveroni, Lo sboom, Silvana, Milano 2009. 
considerazione. Secondo l'ontologia dell'arte di Formaggio, l'arte contemporanea cade in un limbo di incertezza.

\section{Differenza tra arte moderna e contemporanea (1)}

Ma qual è la differenza tra arte moderna e contemporanea?

Se fino agli anni 70-80 del Novecento la distinzione sembrava chiara. Bastava frequentare i più importanti musei di arte moderna come il MOMA di New York per rendersene conto. Era dichiarato, seguendo la tassonomia dei maggiori storici e critici dell'epoca $\left(\mathrm{H}\right.$. Belting) ${ }^{15}$, che l'arte moderna nascesse con Cézanne, Van Gogh e Gauguin, nella seconda metà dell'Ottocento, proseguendo nelle avanguardie storiche. Mentre l'arte così detta "contemporanea" prendeva le mosse, alla fine degli anni Cinquanta del Novecento, sulle orme di Duchamp, con Nouveau réalisme, Pop art, Performance, Arte concettuale, eccetera. Negli ultimi decenni gli storici dell'arte e critici hanno però mischiato l'arte moderna con quella contemporanea, senza rilevare una differenza sostanziale, privata della quale tutto il discorso sull'arte e sul bello cade in confusione.

\section{Arte moderna come riscoperta del bello libero kantiano}

Adorno, tra gli altri, afferma che l'arte moderna, la quale dopo i tre precursori di cui detto, si sviluppa nei primi del Novecento con le avanguardie storiche (astrattismo, cubismo, futurismo, surrealismo, fauve ecc.), abbandona il bello per mettere piuttosto in evidenza contraddizioni e lacerazioni di un'epoca problematica ${ }^{16}$.

Ma se leggiamo i diari e le lettere dei protagonisti di questa modernità artistica scopriamo che non è vero quanto afferma Adorno. Gli artisti

${ }^{15}$ Cfr. H. Belting, La fine della storia dell'arte o della libertà dell'arte, tr. it. di F. Pomarici, Einaudi, Torino 1990.

${ }^{16}$ Cfr. T. L. W. Adorno, Teoria estetica, tr. it. a cura di E. De Angelis, Einaudi, Torino 1977, pp. 78-83. 
modernisti non abbandonano affatto il bello, ma lo riscoprono. Cioè abbandonano il naturalismo, per riscoprire invece il bello originario, ritmicomusicale, ritornando alla "grande tradizione del mondo", in quanto si tratta della bellezza che condividono tutte le culture di tutti i tempi, dall'arte preistorica, a quella egizia, babilonese, greca arcaica, etrusca, precolombiana, piuttosto che africana o orientale ${ }^{17}$. La conferma di ciò è nel fatto che la maggior parte degli artisti modernisti, a cavallo tra Ottocento e Novecento, ha preso come modello proprio le opere di tali culture antiche ed esotiche. $\mathrm{Si}$ tratta di quel bello libero che Kant vede come il bello più autentico, in contrapposizione al bello aderente che nell'ambito artistico possiamo riferire al naturalismo della tradizione europea, che gli artisti, con l'arte moderna, hanno abbandonato ${ }^{18}$. Il bello libero è in effetti per Kant quello che noi oggi, in ambito artistico, chiamiamo "astrattismo". Infatti tra gli esempi di tale bello Kant annovera: la musica senza testo, l'arabesco, la greca, i fiori nel loro insieme (cioè macchie indefinite di colore). L'astrattismo del primo Novecento, per enfatizzare tale bello originario, costituito dalle combinazioni di linee, superfici e colori, elimina la figura. L'origine dell'astrattismo è raccontata da Kandinskij nei suoi diari, quando un giorno entrando nello studio intravide «[...] un quadro indescrivibilmente bello, tutto pervaso di ardore interno. Sulle prime rimasi sbalordito, ma poi mi avvicinai con passo lesto a quel quadro enigmatico, assolutamente incomprensibile nel suo contenuto esteriore, costituito esclusivamente di macchie di colore. E trovai la chiave dell'enigma: era un quadro che avevo dipinto io, appoggiato al muro capovolto [...] $\mathrm{mi}$ fu perfettamente chiaro che l'oggettualità era dannosa ai miei quadri» ${ }^{19}$.

\footnotetext{
${ }^{17}$ Cfr. N. Vitale, Figura Solare, Marietti, Genova-Milano 2011, pp. 69-108.

18 I. Kant, Critica del giudizio, cit., pp. 217 e ss.

19 V. Kandinskij, Testo d'autore e altri scritti russi 1902-1922, tr. it. a cura di C. G. De Michelis, De Donato, Bari 1975, pp. 117, 118.
} 
Se sfogliamo il Cavaliere azzurro, libro scritto a più mani, sorta di manifesto dell'astrattismo, troviamo frequentemente la parola bello, bello eterno come fine dell'arte, contro le convenzioni figurative. Burljùk scrive:

Questi principi costituiscono altrettante fonti inestinguibili di eterna bellezza. Tutti vi possono attingere, solo che abbiano occhi capaci di vedere il senso nascosto delle linee e dei colori. E l'uomo si sente attratto, affascinato, trascinato! 20

Mondrian scrive ad un amico:

Credo sia possibile che, attraverso linee orizzontali e verticali costruite con coscienza, ma non con calcolo, guidate da un'alta intuizione, e portate all'armonia e al ritmo, queste forme basilari di bellezza, aiutate se necessario da altre linee o curve, possano divenire un'opera d'arte, così forte quanto vera ${ }^{21}$.

\section{Differenza tra arte moderna e arte contemporanea (2)}

Se dunque l'arte moderna nasce all'insegna del bello libero cioè della bellezza astratta tra combinazioni di linee, superfici e colori, che scopriamo in tutte le avanguardie storiche (anche dove c'è la figura, in quanto sottomessa ai criteri estetici dell'astrazione), al contrario l'arte contemporanea è fondata sulle intuizioni di Duchamp, sostanzialmente antiestetiche, contro il "carnevale dell'estetismo", ma anche contro l'arte. Duchamp, considerato ispiratore e fondatore dell'arte contemporanea, aveva invece intenzione di distruggere, non il bello, che considerava una forma superficiale di edonismo irrilevante, ma l'arte stessa. Cerca infatti, col ready-made, di dimostrare che l'arte non è fondata su un valore intrinseco dell'opera, ma che si regge sulla vacua suggestione dell'apparato retorico e rituale dell'arte. L'orinatoio e lo scola bottiglie infatti rappresentano per Duchamp oggetti che non esprimono alcuna bellezza o senso, ma che esposti in un museo o in una galleria appaiono

\footnotetext{
${ }^{20}$ V. Kandinskij, F. Marc, Il Cavaliere Azzurro, tr. it. di R. Calzecchi Onesti, De Donato, Bari 1967, p. 46.

${ }^{21}$ P. Mondrian, Lettera del 1914 a Hans-Peter Bremmer, in M. Seuphor, Piet Mondrian. La vita e l'opera, Il Saggiatore, Milano 1962.
} 
come oggetti misteriosi, acquisendo un senso falsamente simbolico, in cui, per il francese, risiede il senso stesso dell'arte, fondato sull'ingenuità di suggestioni vacue. Arriva ad affermare: (L'arte) «è qualcosa che appartiene alla sfera della masturbazione» ${ }^{22}$, quindi dichiara drasticamente: "potremmo creare una società che rifiuti l'arte» ${ }^{23}$, in quanto la considerava una sorta di inganno. Affermazione che richiama singolarmente i precetti posti da Platone ne La Repubblica per salvaguardare il governo della ragione ${ }^{24}$.

Non ci dilungheremo sui motivi per cui Duchamp compie tale operazione radicalmente distruttiva, (motivi complessi e reconditi che si annidano in problemi psicologici in relazione alla sua famiglia, dove l'arte era stata mitizzata) ${ }^{25}$. Tuttavia esiste una lettera (che nel mondo dell'arte contemporanea non è mai stata pubblicata per intero) significativa al riguardo, che mette in evidenza il totale fraintendimento o falsificazione nei riguardi della sua intuizione del ready-made, che $\mathrm{i}$ primi artisti contemporanei operarono. La lettera è la seguente:

\footnotetext{
Questo Neo-Dada che viene chiamato ora: Nouveau Réalisme, Pop Art, Assemblaggio, ecc., è una distrazione a buon mercato che vive di quello che Dada ha fatto. Quando ho scoperto il ready made, speravo di scoraggiare il carnevale dell'estetismo. Ma i neo-dadaisti utilizzano il ready made per scoprirvi un valore estetico. Gli ho tirato in testa il portabottiglie e l'orinatoio come una provocazione ed ecco che loro ne ammirano la bellezza estetica ${ }^{26}$.
}

Duchamp disprezzava i nuovi artisti di arte contemporanea, che lo vedevano come un ispiratore, ma che secondo il francese non avevano capito il suo gesto distruttivo, trasformandolo in un gesto costruttivo. Ma proprio in quella commutazione, in quel cambio di segno, per cui si tenta di ripristinare un valore estetico da un atto di azzeramento nichilista, è il bug dell'arte

\footnotetext{
${ }_{22}$ M. Duchamp, Ingegnere del tempo perduto. Conversazione con Pierre Cabanne, tr. it. di W. Marchetti, Multhipla Edizioni, Milano 1979, p. 145.

${ }^{23}$ Ibidem.

${ }^{24}$ Cfr. Platone, La Repubblica, Rizzoli, Milano 1953, Libro X, 596a ss.

${ }^{25}$ N. Vitale, Figura Solare, cit., pp. 220-232.

${ }^{26}$ M. Duchamp, Lettre du 10 novembre 1962, in H. Richter, Dada-art et anti-art, Bruxelles, Édition de la Connaisance, 1965, p. 196.
} 
contemporanea, l'anello debole della catena. Ed è esattamente quello che denuncia Duchamp: l'abuso nell'avere cercato il bello in un orinatoio e in uno scola bottiglie, dove non c'è nulla di bello, egli infatti li vedeva piuttosto come oggetti contundenti da "tirare in testa" al pubblico come provocazione.

Il paradosso dell'arte contemporanea e il suo punto debole, sta proprio nell'aver sfruttato, come aveva intuito Duchamp, l'apparato retorico del sistema dell'arte per enfatizzare cose del tutto prive di senso. Ma il francese voleva, con questo, smascherare quella che secondo lui è la farsa dell'arte, mentre gli artisti dell'arte contemporanea (che Duchamp chiama neodadaisti) sfruttano lo stesso principio per rifondare l'arte al di fuori dei canoni estetici tradizionali (antichi e moderni). È possibile così credere che tali oggetti, una volta esposti in un museo, abbiano un valore estetico intrinseco, mentre non lo hanno, sdoganati come arte per un groviglio di equivoci.

\section{Tutta l'arte è contemporanea?}

Oggi va di moda ripetere una proposizione che gira malauguratamente nelle accademie e nelle università da un certo tempo: «tutta l'arte è contemporanea", di cui francamente non conosco l'origine. Frase che vorrebbe mettere in discussione l'attribuirsi, da parte della così detta arte contemporanea odierna, in virtù del suo carattere sperimentalista, il fregio di primato del senso, rispetto ad altre tendenze dell'arte di oggi. Si contesta infatti che il concetto stesso di "arte contemporanea" possa contrassegnare un particolare statuto ontologico delle opere e in un certo senso dargli un valore di attualità. Al contrario, affermando "tutta l'arte è contemporanea", si rivendica che tale accezione possa riferirsi, non solo a tutta l'arte prodotta oggi, ma a tutta l'arte di tutti i tempi, in quanto sempre prodotta (a suo tempo) nel proprio tempo, e dunque appunto: arte contemporanea. 


\section{Cos'è l'arte contemporanea?}

Sembra che il significato di arte contemporanea in questi anni sia stato dimenticato, che, al contrario di quanto vuole sostenere tale critica implicita, indica proprio uno specifico statuto ontologico, per cui non tutta l'arte è "arte contemporanea".

Devo ammettere che a ragguagliarmi sulla questione è stato Pierre Restany, con cui ho avuto a che fare per un certo periodo negli anni 90. Il critico francese è stato l'ideologo del Nouveau Réalisme, il primo movimento di arte contemporanea, seguito a ruota, qualche mese dopo, dalla Pop art. Infatti Nouveau Réalisme e Pop art, soprattutto nei loro retroscena funzionali e ideologici, rappresentano il nucleo dualistico su cui si fonda l'arte contemporanea, le due colonne portanti che la reggono. Se Pierre Restany è stato il teorico che ha contribuito a forgiare l'idea di arte contemporanea, il suo supporto scientifico, dall'altra la Pop art era proposta da Leo Castelli, un gallerista di origini italiane, naturalizzato americano, che ha forgiato a sua volta l'aspetto logistico della stessa, potenziando quello che oggi viene chiamato "sistema dell'arte". Una organizzazione a più livelli promozionali, che ha coinvolto il jet-set internazionale tra attori, cantanti pop, stilisti di alta moda, yuppie della new economy e della borsa di Wall Street, oltre ai già presenti imprenditori e borghesi di alto profilo. Per sostenere la nuova arte, che non ha più un valore intrinseco fondato tradizionalmente sul bello, occorreva una struttura teorica e un'impalcatura promozionale che motivasse ed enfatizzasse le opere per dargli credibilità. Struttura che si è infine consolidata nel mercato, in particolare nelle aste che sanciscono ufficialmente il valore delle opere. Un mercato specialistico che pervade il sistema e lo alimenta, enfatizzandolo senza precedenti, arrivando a speculazioni vertiginose, dove le singole opere vengono pagate fino a svariati milioni di dollari. 
Dunque, secondo Pierre Restany, “arte contemporanea” non è, come molti pensano, un termine generico, che si riferisce a un'arte fatta oggi, dai contemporanei, accezione che sarebbe del tutto banale, ma si tratta di un termine tecnico, specifico, che designa un'arte che non poteva essere realizzata prima di quel preciso momento, cioè che non può prescindere dalle elaborazioni precedenti. Un'arte che si costituisce come un sempre ulteriore passo innovativo dell'elaborazione del linguaggio, passo consequenziale e necessario. È un'arte dunque che si fonda su un paradigma storico-critico ben strutturato, che sancisce a priori cosa sia linguaggio artistico e come si articoli nei suoi modi di sviluppo. Quindi un artista di arte contemporanea non può più pensare come un artista tradizionale, alla ricerca libera dell'espressione di sé, ma deve pensare come un critico. Deve conoscere, non solo il modo con cui i materiali e gli oggetti possano diventare direttamente segni e linguaggio, ma, se vuole realizzare un'opera valida, deve conoscere quanto è stato precedentemente realizzato nello stesso ambito, per potersi inserire con la sua opera in un processo in divenire, dalla struttura precostituita. Dunque il senso delle opere di arte contemporanea è interamente temporalizzato, giustificato dal paradigma storico critico, così come è legittimato dal sistema di enfatizzazione e di mercato. Ma ciò nasconde una inconcepibile contraddizione in quanto il primo è fondato sulla denuncia della civiltà contemporanea con un’impronta etica e politica, l'altra al contrario è l'espressione del capitalismo vigente, criticato dalla prima. D'altro canto, senza entrambe queste forze, l'arte contemporanea non avrebbe senso e rilevanza, in quanto, come dimostrato, dopo sessant'anni dalla sua nascita, non viene riconosciuta dal vasto pubblico. In ciò è il suo tallone d'Achille, per cui con la crisi delle ideologie ed economica si è innescato un processo entropico che è destinato nei prossimi anni ad accelerarsi in modo esponenziale, che vedrà il sistema dell'arte contemporanea implodere e le opere svanire progressivamente con lo svanire della stessa suggestione che le reggeva. 


\section{Un ritorno al bello?}

La distinzione tra arte moderna e contemporanea vede dunque la prima abbandonare il naturalismo e il relativo bello aderente, per riscoprire il bello libero; la seconda abbandonare anche il bello libero, per centrare il senso sul divenire sperimentalista del linguaggio.

La questione rilevante che abbiamo osservato: quando l'arte abbandona il bello (aderente e libero) non viene più seguita dal pubblico. Se ne deduce che il più vasto pubblico, nonostante la gran confusione registrata, considera l'arte indissolubilmente legata al bello. Da qui possiamo trarne le dovute conclusioni in merito alla teoria di Formaggio inerente il criterio ontologico dell'arte (considerando che quando l'arte si discosta dal bello gli uomini non continuano a chiamarla arte), cioè che, come afferma Severino, arte e bellezza sembrano legate necessariamente.

Per questa ragione la decadenza conclamata dell'arte contemporanea pone evidentemente l'ipotesi che, dopo questa parentesi di un'arte intellettualizzata e così allontanatasi dalla sua essenza, l'arte debba necessariamente ritrovare il suo fondamento originario. Ma sorge spontanea la domanda: se l'arte dovrà recuperare il bello, ciò dovrà avvenire necessariamente ritornando ai vecchi valori metafisici? Cosa che sconcerta i filosofi che sentono questo come un ritorno a un passato ormai inaccettabile per le ragioni che conosciamo.

Non stupisce che dal punto di vista intellettuale la questione del bello, in filosofia, venga cercata necessariamente a partire proprio da Platone, come colui che ne ha consolidato la visione metafisica, per cui i valori universali sono concatenati nell'Idea tra Bello, Buono, Vero. Platone è d'altro canto il filosofo tra i primi ad affermare il distacco tra il bello e l'arte, attribuendole una pura funzione mimetica ${ }^{27}$. Tuttavia è evidente che la messa in

${ }^{27}$ Platone, La Repubblica, cit., Libro X, 596a ss. 
discussione di tale prospettiva filosofica non può venire dalla filosofia, che cerca inevitabilmente di fondare il senso su un paradigma intellettuale. Ma dovrebbe poter emergere dall'esperienza di coloro che hanno la sensibilità per orientarsi in un altro percorso di conoscenza. Forse per questo Wittgenstein affermava che dell'arte non se ne dovesse parlare, per lasciare interamente le sue sorti in mano a chi la pratica. Ma come abbiamo già osservato al giorno d'oggi, l'esperienza del bello e dell'arte, al di là della filosofia e della critica, per diverse ragioni, non del tutto imputabili al mondo intellettuale, sono entrambi frammentati e frammentari, caduti nel relativismo più estremo.

Se torniamo ad Adorno, alla sua affermazione che l'arte moderna si distacca dal bello, possiamo forse chiarire l'equivoco. È evidente che Adorno considera il bello relativo alla tradizione naturalistica, cioè sembra riferirsi all'esperienza esistenziale più comune del bello, per cui lo riconosciamo in un uomo, una donna, un cavallo, una casa, eccetera. Come abbiamo rilevato si tratta dunque del bello aderente kantiano. $\grave{\mathrm{E}}$ questo un problema di percezione: gran parte degli intellettuali, non sensibili al bello astratto (bello libero), fanno del bello aderente, come esperienza comune, il "bello" tout court, pensato all'ingrosso come una delle tante categorie della rappresentazione (insieme al brutto, sublime, kitsch, comico, orrendo, eccetera), come se tale bello potesse essere un'evidenza, allontanandosi così dall'accezione del bello come valore estetico e specifica conoscenza dell'arte, creando un equivoco tanto banale, quanto imbarazzante. Infatti tutti sappiamo, con un'evidenza che non ha bisogno di giustificazioni, che la bellezza o meno di un quadro o di una scultura, prescinde da ciò che vi è rappresentato, che sia una bella figura o mostruosa. Cioè il Saturno che divora $i$ suoi figli, di Goya, è un bellissimo dipinto, così come lo è la Maya denuda, mentre la Maya vestida, dello stesso Goya, è un quadro che, pur raffigurando una bella ragazza, possiamo dire che è, se non proprio un brutto quadro, esteticamente abbastanza scadente, del tutto manierato, come risalta dal confronto col quadro gemello. 


\section{Differenza tra bello libero e bello aderente}

Occorre ora interrogarsi sulla differenza sostanziale tra bello aderente e bello libero. Il bello aderente, che nell'arte visiva caratterizza la tradizione naturalistica europea, riferendosi a un concetto è ascrivibile proprio a quella concezione metafisica del bello (Platone) tanto contrastato dalla cultura novecentesca. Al contrario il bello libero, che caratterizza non solo l'arte moderna, ma tutta l'arte non naturalistica (astratta e figurativa) ${ }^{28}$, lo si può considerare il bello non metafisico, in quanto costituito da armonie empiriche di forme e colori, che abbiamo ipotizzato costituirsi come l'essenza stessa dell'arte.

Stiamo toccando il punto cruciale di tutto il nostro discorso. Se dunque, secondo una statistica che segue l'ontologia di Formaggio, il bello libero costituisce l'essenza dell'arte (in quanto la maggior parte di arte in una panoramica universale non è naturalistica), prima ancora di considerare la questione del "bello" in generale, ne dovremmo trovare il fondamento in una struttura concreta, condivisa, dell'arte, che sia riconoscibile come comune denominatore, al di là delle differenze di epoche e culture. A questo punto la domanda cruciale è: esiste una costante antropologica dell'arte? Se in uno sguardo a volo d'uccello osserviamo tutte le culture in tutti i tempi, possiamo

${ }^{28} \mathrm{Il}$ bello libero kantiano può essere, nell'arte visiva, astratto (Kant lo riferisce alla musica senza testo, all'arabesco e alla greca) o figurativo (Kant lo riferisce ai fiori nel loro insieme, che non sappiamo come devono essere fatti). Tuttavia Kant non si interroga a fondo da cosa sia costituita la suggestione e il piacere del bello libero, riferendolo come nel caso del bello aderente, a un'idea estetica, in questo caso di tipo «musicale». Diversamente se avesse potuto accertarsi che il bello libero si fonda sulla percezione di armonie costituite da frequenze elettromagnetiche del campo sonoro come di quello visivo, che creano assonanze o dissonanze (in fisica interferenza costruttiva e distruttiva) avrebbe rilevato che quella bellezza non dipende da una "idea estetica", e che qualsiasi tipo di forma non determinata può assumere quelle armonie interne, risultando, alla sensibilità, belle. Pervenendo a questa concezione del bello libero Kant non avrebbe avuto bisogno del concetto di sublime per giustificare esteticamente la rappresentazione del brutto, orrendo o terribile, in quanto figure che possono essere come qualsiasi altra armonizzate in una forma di splendore costituito da armonie astratte, come appunto vediamo nel Saturno di Goya, un dipinto "bello", indipendentemente dal fatto che il suo contenuto possa essere ritenuto "sublime". 
forse trovare il trait d'union. In quanto l'arte, vista da questa prospettiva, pare sempre implicata, sin dalla sua origine, in una efficacia estatica-estetica che possiamo cercare di chiarire.

\section{Una fisiologia dell'arte}

È un'idea di Nietzsche: una "fisiologia dell'arte"29. Il filosofo tedesco, usando il metodo genealogico, cerca l'origine delle cose in esigenze pratiche finalizzate innanzitutto alla sopravvivenza ${ }^{30}$. Se infatti riusciamo a tornare a quell'origine, scopriamo che l'arte ha una funzione specifica, associata a magia e religione, da cui nasce come strumento liturgico. Un breve excursus sull'origine fisiologica dell'arte, sulla sua funzione primordiale, non è un discorso nuovo, ma indispensabile a richiamare alla mente una questione essenziale, per rendersi conto di qualcosa che, se analizzato nei suoi processi funzionali, appare ovvio.

Il linguaggio discorsivo, da cui sorge il pensiero razionale, presentando alla coscienza le cose senza la loro presenza fisica, scinde il concetto dalla sensorialità, divide la coscienza e, di conseguenza, la percezione del mondo. Questa scissione ha vaste conseguenze sulla coscienza umana. Specchiandosi nel linguaggio l'uomo conquista un'autocoscienza, il cui effetto collaterale è l'angoscioso sentimento di distacco dalla natura e consapevolezza della finitudine, che a sua volta porta a una necessità reintegrativa. Il rito estatico primordiale ha la funzione di reintegrare quella scissione, stemperando la coscienza razionale, per privilegiare la percezione di una pienezza riconquistata. Attraverso canti e danze, i primi uomini possono reimmergersi nell'ordine naturale enfatizzando l'aspetto energetico corporeo. Occorre rilevare che l'uomo non può vivere senza questo antidoto agli effetti collaterali

\footnotetext{
${ }^{29}$ Cfr. M. Heidegger, "La volontà di potenza come arte", in Nietzsche, cit., pp. 21-215.

${ }^{30}$ Cfr. F. Nietzsche, Umano, troppo umano, tr. it. a cura di G. Colli e M. Montanari, Adelphi, Milano 1965, 1979.
} 
dell'autocoscienza. Antidoto che prende forma all'origine in una proto-arte che si costituisce come strumento liturgico di magia e religione. Assumendo nei secoli una progressiva autonomia, le arti focalizzano la funzione reintegrativa sul linguaggio simbolico, sfaccettandolo in una sempre più differenziata polivalenza. Ad esempio, per quanto riguarda l'arte visiva, i piani principali sono: la sensorialità con cui è plasmata la materia, la sintassi cromatica, la definizione della forma della rappresentazione legata ad un concetto, il sentimento emerso dai caratteri psicologici delle figure rappresentate e dalle atmosfere degli sfondi, i simboli, i contenuti narrativi mitologici, la razionalizzazione dello spazio con la prospettiva e la teoria delle ombre. Se dunque l'aspetto mistico dei rituali originari riavvicina all'unità dell'essere annullando le differenziazioni della coscienza, l'arte invece lo fa "rimontandone" i frammenti uno sull'altro, come in un gioco di costruzioni. Per fare ciò occorre non solo un affastellamento dei piani diversi del linguaggio espressivo (descritti), ma una loro coessenzialità in una unità sensibile, realizzata con le armonie formali del ritmo. Così facendo l'arte ricostruisce l'esperienza dell'essere, diremmo, "in vitro", cioè riproduce l'organicità della vita filtrata nel linguaggio espressivo. In questo si costituisce come un potente antidoto agli effetti collaterali della scissione originaria, pur mantenendo in parte la differenziazione della coscienza nei vari piani del linguaggio simbolico, senza riportarli all'indistinto come nelle pratiche reintegrative primordiali.

Se osserviamo tutta l'arte di tutti i tempi scopriamo che è realizzata allo stesso modo: un linguaggio polivalente, più o meno sfaccettato e differenziato, tenuto insieme da una forza ritmica. Concetto e materia, contenuti e forme, assumono in questa coordinazione reciproca un senso sovrastorico, in virtù della forza attiva che li lega, senso che chiamiamo "bello". Diversi filosofi si sono avvicinati a questa visione fisiologica dell'arte coi concetti di unità nella varietà (Diderot), con le categorie di aorgico e organico (Hölderlin), di dionisiaco e apollineo (Nietzsche) astrazione e empatia (Worringer). 
Osserviamo come in questa visione funzionale dell'arte il concetto di arte e di bello si fondono, in quanto l'arte diventa tecnica di quell'estasi riunificante originaria.

Il piacere del bello è prodotto infatti dal percepire piani di coscienza che consuetamente viviamo separatamente, unificati in armonia e coessenzialità.

Questa concezione organica del bello e dell'arte non ha nulla a vedere con una concezione ideale metafisica, è un valore empirico che nasce dalla pratica di conciliazione di elementi ontologicamente diversi che fanno capo a funzioni diverse della coscienza: razionale, intuitiva, affettiva, sensoriale. In questa prospettiva si giustifica il senso universale dell'arte in quanto, come osservato, gli elementi del linguaggio, pur storicamente determinati, entrando in relazione tra loro, assumono un senso diverso, che trascende il linguaggio stesso e la sua storicità: il bello artistico.

\section{La funzione del canone}

Per concludere occorre approfondire la questione dell'universalità del bello in confronto con il relativismo filosofico che tende a tacciare tale concezione di platonismo, romanticismo o metafisica. Questa obiezione è d'altro canto comprensibile, in quanto se pensiamo a quanto sostiene Kant, che la percezione del bello, affidata al sentimento, sia uguale per tutti gli uomini, attribuiamo al sentimento una facoltà universale ormai messa in discussione non solo da confutazioni teoriche, ma anche dalle molte esperienze che in più frangenti e in più ambiti, ci troviamo ad affrontare. Insomma il romanticismo è dietro l'angolo, quel romanticismo che pretende di poter determinare contenuti e forme assoluti. Oggi posizione difficile da accettare.

Per venire a capo della questione occorre considerare, come dicevamo all'inizio, che oggi le arti sono state svincolate da ogni canone, proprio per abbandonare ogni pretesa di universalità dei modelli predeterminati. L'azione valutativa del sentimento, liberata dai modelli, si trova così persa in 
troppe forme e suggestioni, slegate le une dalle altre, suggestioni alle quali il sentimento aderisce con difficoltà, spesso momentaneamente, nella tipica volubilità della soggettività che oggi più che mai appare priva di alcuna valenza universale.

Oggi gli artisti possono ritenersi liberi di esprimersi come vogliono, ma stanno scoprendo che ciò che possono esprimere non è affatto condivisibile, cioè sono sì liberi, ma soli e impotenti: nessuno li ascolta. In tale pretesa libertà di espressione, oggi, avendo perso ogni universalità, per "universalizzare" l'espressione artistica, ci si deve rimettere a ciò che può essere riconosciuto socialmente. Insomma, per poter essere apprezzati come artisti, una volta dismessi i canoni, occorre seguire le mode culturali, le tendenze di mercato, sfruttare tematiche sociologiche sensibili, affiliarsi a poteri che possano proteggere e garantire, promuovere il proprio lavoro e la propria immagine in ogni modo, diffondere il nome. La libertà di espressione, privata di universalità, si volta nel suo contrario, in un estremo condizionamento superficiale.

D'altro canto, come osservato, i canoni delle varie tradizioni sono visti come costrizioni che hanno avuto la pretesa di fondare un senso universale a priori, cadendo anche in questo caso in una forma metafisica inaccettabile. Ma anche qui occorre una distinzione in quanto, questo è vero per i modelli naturalistici in genere e i canoni antropometrici della tradizione classica, ripresi nel Rinascimento, che facevano capo alla bellezza del corpo umano fissata matematicamente, cioè al bello aderente. Mentre non lo è per quanto riguarda i canoni medievali, arcaici ed orientali che facevano capo al bello libero, che come osservato non risente del vizio metafisico. In questo modo il canone si presenta sotto tutt'altra luce. Florenskij ha affrontato questa questione in modo originale nel suo famoso testo sull'icona bizantina:

[...] l'esigenza della forma canonica, [...] è una liberazione e non una limitazione. L'artista il quale per ignoranza si immagina che senza una forma canonica creerebbe qualcosa di grande, somiglia al viandante cui sembri d'ostacolo il 
terreno e s'immagini che appeso per aria andrebbe più lontano che per terra. Un artista che abbia ripudiato l'integra forma, inconsapevolmente si afferra ai residui e ai lembi di quella forma, casuali e incompleti e per queste inconsapevoli reminiscenze pretende l'epiteto di 'creazione'. Viceversa, l'autentico artista non vuole la cosa sua a ogni costo, ma vuole il bello, l'oggettivamente bello, cioè l'artistica configurazione della verità delle cose e in genere non si cura della questione meschina e vanitosa, se è il primo o il centesimo a parlare della verità. Basta che essa sia la verità, e il valore dell'opera è garantito. Chi vive si preoccupa di vivere veracemente e non se la sua vita è simile a quella del vicino, vive per conto suo per la verità ed è convinto che una vita sincera per la verità è comunque individuale e nella sua essenza del tutto irripetibile, e può essere vera soltanto nel flusso della storia universale dell'umanità e non come deliberata invenzione - così la vita artistica: anche l'artista, appoggiandosi ai canoni artistici universalmente umani, che si ritrovano tali e quali qui e altrove, per mezzo di essi e in essi trova la forza di configurare la realtà autenticamente contemplata e sa con certezza che la sua opera, se è libera, non risulta un duplicato dell'altrui, benché l'oggetto della sua preoccupazione non sia questo confronto con qualcun altro, ma la verità di ciò che raffigura ${ }^{31}$.

Il punto di vista di Florenskij è evidentemente opposto al relativismo filosofico contemporaneo dal quale è tacciato di neoplatonismo. A parte questa questione che può essere discussa ${ }^{32}$, Florenskij, nella sua interpretazione dell'icona bizantina, vede il canone predisposto affinché si riproduca, in una sorta di ritualità formale, quella organicità dell'opera necessaria e funzionale al suo fine estetico atemporale, molto simile come vedremo all'attuale esecuzione strumentale della musica classica, che si fonda su un canone. Ma cerchiamo di comprendere meglio di cosa si tratta:

[...] tutti questi e simili caratteri della superficie dell'opera, come l'estensione, la fermezza, si comunicano alla fattura dell'opera e creano i loro equivalenti dinamici, cioè da latenti, passivi, immobili, si trasformano in sorgenti di forza e penetrano nell'ambiente circostante. Come il polo di forza invisibile del magnete diventa visibile per mezzo della limatura di ferro, così la struttura statica della superficie si manifesta dinamicamente mediante il colore sparso sulla superficie stessa, e quanto più perfetta è l'opera d'arte figurativa, tanto più è evidente questo manifestarsi. Acuta è la mente che risiede nelle dita e nella mano dell'artista, questa mente acuta capisce, senza bisogno dell'intelligenza di testa, l'essenza di tutti questi rapporti di forza della superficie della rappresentazione, e penetra a fondo queste essenze, riconoscendo, se il materiale fu scelto come si

${ }^{31}$ P. Florenskij, Le Porte Regali, tr. it. a cura di E. Zolla, Adelphi, Milano 1977, pp. 79, 80.

${ }^{32}$ La terminologia di Florenskij, in particolare, in questo caso, in accezioni come "bello oggettivo", denuncia il suo neoplatonismo, che a tratti è evidente, ma il suo pensiero per certi aspetti richiama invece Simmel o Dilthey, in quella sua assidua contaminazione fra filosofia come scienza rigorosa e sapienza, pur nella forma espositiva evidentemente dissimile dai due filosofi. 
deve, in esse e nel loro corrispettivo problema stilistico, una particolare struttura spirituale [...]. Avendo penetrato la struttura della superficie, l'intelligenza manuale la manifesta nella fattura del suo tocco. Perciò tutta la concezione dell'artista è stilisticamente conforme al materiale; e se non è conforme, è predestinata a esserlo dalla natura delle cose, quando con l'intelligenza delle dita l'artista identifica la superficie, viene escludendo come sconveniente tutto ciò che ad esso è estraneo ${ }^{33}$.

Il canone non naturalistico (non metafisico) permette di impostare una relazione tra pratiche artigianali e sensibilità astratte, tra determinazioni razionali e stati affettivi spazializzati. Induce a vedere nelle forme le loro relazioni, un percorso indispensabile per quel salto di qualità, che non è solo un'abilità tecnica, ma è una presa di coscienza e una rivelazione nella percezione del bello libero. L'espressione individuale in questo caso non è più legata alla scelta soggettiva di forme e contenuti, ma alla intensità ed essenzialità con cui vengono coordinati nelle relazioni sensibili.

Ciò lo possiamo cogliere in modo evidente nell'esecuzione della musica classica, dove esercizi, in un primo tempo meccanici, diventano progressivamente gli strumenti di una liberazione, in quanto l'artista esecutore concentra tutta la sua creatività nel coordinare in un modo del tutto originale, quei piani espressivi studiati separatamente. Entrando così in uno stato che trascende sia la sua soggettività sia la musica scritta, quel bello musicale costituito da entrambe, in una compresenza superiore.

Questo percorso complesso nel seguire un canone, costituisce il processo per cui la soggettività assurge a un valore universale non metafisico. Generalizzando si tratta proprio di quel punto così controverso per cui il sentimento, applicandosi assiduamente alle stesse forme in tecniche di coordinazione, assume una straordinaria sensibilità e accede a una dimensione di intersoggettività. Il valore, in questo caso, non è nella determinazione di un linguaggio, dunque legata a un'idea di forma, contenuto, stile, ma è nella sapienza e originalità di coordinazione dei piani

${ }^{33}$ Ivi, pp. 111, 112.

Materiali di Estetica - N. 8.2: 2021, Pagina 403 
espressivi. In questo modo non conta più se l'opera si instaura in un progetto in divenire, volto alla ricerca del "nuovo", o si costituisce su forme consolidate nella tradizione, in quanto il suo rinnovamento avviene dall'interno. Come nell'esecuzione della musica classica, non conta se il brano sia stato eseguito molte volte, conta l'originalità e intensità dei modi con cui è ogni volta rielaborato nella sua polivalenza in un delicato equilibrio. Ma se usiamo un linguaggio che, come nell'arte contemporanea, non è predisposto per accogliere quella polivalenza e unità, non potremo realizzare un'opera che trascende il linguaggio, ma avremo solo un tentativo mancato, un'opera che non può arrivare alla sua realizzazione universale, ma rimane relegata ai vari piani immanenti del linguaggio, e ai loro significati particolari, cosa che la accomuna a qualsiasi manifestazione non artistica.

Dunque il sentimento, per poter percepire ed esprimere una valenza universale, deve necessariamente applicarsi al processo disciplinato previsto dal canone, in quanto il canone è strutturato organicamente, diventando un aiuto indispensabile per comprendere la struttura polivalente dell'arte. Canone che, una volta utilizzato per acquisire sensibilità e padronanza dell'essenza dell'opera d'arte, può essere superato, come vediamo negli artisti moderni delle avanguardie storiche che, nei primi del Novecento, assimilano in un primo momento i canoni di tradizioni antiche ed esotiche, per, una volta afferrata la struttura polivalente, fondare un metodo personale che diventa una sorta di canone individuale. Nietzsche scrive: «[...] attraverso l'esercizio e un modello noi diventiamo noi stessi!» ${ }^{34}$.

${ }^{34}$ F. Nietzsche, Opere di Friedrich Nietzsche, a cura di G. Colli e M. Montinari, Adelphi, Milano 1964 sgg., vol. V, t. II, Frammenti Postumi 1879-81, p. 560. 


\section{Nota bibliografica}

ADORNO, Theodor Ludwig Wiesengrund, Teoria estetica, tr. it. a cura di E. De Angelis, Einaudi, Torino 1977.

BELTING, Hans, La fine della storia dell'arte o della libertà dell'arte, tr. it. di F. Pomarici, Einaudi, Torino 1990.

DUCHAMP, Marcel, Lettre du 10 novembre 1962, in H. Richter, Dada-art et anti-art, Bruxelles, Édition de la Connaisance, 1965.

—, Ingegnere del tempo perduto. Conversazione con Pierre Cabanne, tr. it. di W. Marchetti, Multhipla Edizioni, Milano 1979.

FLORENSKIJ, Pavel, Le Porte Regali, tr. it. a cura di E. Zolla, Adelphi, Milano 1977.

FORMAGGIO, Dino, L'arte come idea e come esperienza, Mondadori, Milano 1981, poi Morcelliana, Brescia 2018.

HEIDEGGER, Martin, Nietzsche, tr. it. a cura di F. Volpi, Adelphi, Milano 1994.

KANDINSKIJ, Vasilij, Testo d'autore e altri scritti russi 1902-1922, tr. it. a cura di C. G. De Michelis, De Donato, Bari 1975.

KANDINSKIJ, Vasilij, MARC, Franz, Il Cavaliere Azzurro, tr. it. di R. Calzecchi Onesti, De Donato, Bari 1967.

KANT, Immanuel, Critica del Giudizio (1790), tr. it. a cura di L. Amoroso, Rizzoli, Milano 1995.

MONDRIAN, Piet, Lettera del 1914 a Hans-Peter Bremmer, in M. Seuphor, Piet Mondrian. La vita e l'opera, Il Saggiatore, Milano 1962. 
MUNARI, Bruno, Munari 80: a un millimetro da me. Teoremi, brustoline e disegni al telefono, Scheiwiller, Milano 1987.

NIETZSCHE, Friedrich, "Umano, troppo umano", in Opere di Friedrich Nietzsche, a cura di G. Colli e M. Montinari, vol. IV, t. II, Adelphi, Milano 1964 sgg.

—, "Frammenti Postumi 1879-81", in Opere di Friedrich Nietzsche, a cura di G. Colli e M. Montinari, vol. V, t. I, Adelphi, Milano 1964 sgg.

PERNIOLA, Mario, L'arte espansa, Einaudi, Torino 2014.

PLATONE, La Repubblica, Rizzoli, Milano 1953.

POLVERONI, Adriana, This is contemporary! Come cambiano i musei d'arte contemporanea, FrancoAngeli, Milano 2007

—, Lo sboom, Silvana, Milano 2009.

SCARAMUZZA, Gabriele, "Dino Formaggio: una presentazione", Eikasia, Revista de Filosofía, lxii, 2015, pp. 13-28.

SEVERINO, Emanuele, Il bello, Mimesis, Milano-Udine 2011.

STEINER, George, Vere presenze, tr. it. di C. Béguin, Garzanti, Milano 1992.

VALERY, Paul, Discours sur l'esthétique, in Euvres, Gallimard, Paris 1975-1977.

VICO, Giambattista, De antiquissima Italorum sapientia (1710), in Opere filosofiche, a cura di P. Cristofolini, Firenze, Sansoni 1971.

VITALE, Nicola, Figura Solare, Marietti, Genova-Milano 2011.

WITTGENSTEIN, Ludwig, Tractatus logico-philosophicus, tr. it. a cura di G. C. M. Colombo, Fratelli Bocca Editori, Roma-Milano 1954. 


\section{Nota biografica}

Nicola Vitale (Milano, 1956) è poeta, pittore e saggista. Presente alla $54^{\circ}$ edizione della Biennale di Venezia (Padiglione Italia). Ha pubblicato diverse raccolte di poesia in prevalenza nella collana "Lo Specchio" di Mondadori. È presente nelle antologie nazionali di poesia di Mondadori e Bompiani. Ha pubblicato diversi saggi di estetica con le case editrici Marietti, Moretti e Vitali, Mimesis. Collabora dal 2001 con la Casa della Cultura di Milano, con relazioni inerenti l'arte visiva. Ha pubblicato alcuni saggi di estetica sulla rivista «Itinera» dell'Università degli Studi di Milano. Nel 2014, nell'ambito della cattedra di Filosofia del Professor Giordanetti, presso l’Università degli Studi di Milano, è stata discussa una tesi di laurea inerente una tesi originale trattata nel suo saggio Figura Solare (Marietti, Genova-Milano 2011): G. M. Bevacqua, Dalla Nascita della Tragedia, una "configurazione esplicita” del dualismo apollineo e dionisiaco in un interpretazione attuale). 